\title{
El Papel de las Representaciones Sociales en el Maltrato Infantil y en los Procesos de Crianza: una Perspectiva Educativa
}

María Laura Yepes Escobar ${ }^{1}$; De La Rosa-Isaza, Jasson Alberto ${ }^{2} \&$ Parra-Bolaños, Nicolás $^{3(*)}$

${ }^{1}$ Universidad de San Buenaventura, Medellín, Colombia.

2 Institución Universitaria Digital de Antioquia, Rectoría, Medellín, Colombia.

${ }^{3}$ Asociación Educar para el Desarrollo Humano, Laboratorio de Neurociencias y Educación, Buenos Aires, Argentina.

\section{RESUMEN}

El presente estudio de investigación cualitativa parte de una serie de análisis discursivos en el marco de un proceso conceptual que permitió ver la configuración entre creencias y representaciones de carácter social alrededor del fenómeno del maltrato infantil en la crianza de los niños y niñas y dentro del marco educativo contemporáneo. El enfoque gnoseológico de esta investigación tuvo como esencia el interaccionismo simbólico, por lo cual las estrategias metodológicas para el análisis son los grupos de discusión, la entrevista a profundidad y la observación participante, al posibilitar la emergencia de las representaciones sociales a través de la interacción y conversación con padres de familia. Este estudio ha encontrado que, los niños y adolescentes también están en capacidad de llevar a cabo análisis profundos sobre las situaciones de violencia, y se recomienda que dentro del sistema educativo colombiano y latinoamericano, pueda proporcionársele a niños, adolescentes y jóvenes, los contextos enriquecedores necesarios en materia afectiva, emocional, intelectual y relacional para que puedan desarrollarse de forma equilibrada, saludable y adaptativa.

Palabras clave: Representaciones sociales; Maltrato infantil; Crianza. Familia; Educación

\section{The role of social representations in child abuse and in parenting processes: an educational perspective}

\section{ABSTRACT}

The present qualitative research study is part of a series of discursive analyzes within the framework of a conceptual process that allowed us to see the configuration between beliefs and representations of a social nature around the phenomenon of child abuse in the upbringing of children and within the framework contemporary educational. The gnoseological approach of this research had symbolic interactionism as its essence, for which the methodological strategies for analysis are discussion groups, in-depth interviews and participant observation, by enabling the emergence of social representations through interaction and conversation with parents. This study has found that children and adolescents are also able to carry out in-depth analyzes of situations of violence, and it is recommended that within the Colombian and Latin American educational system, children, adolescents and young people can be provided with enriching contexts. Necessary in affective, emotional, intellectual and relational matters so that they can develop in a balanced, healthy and adaptive way.

Keywords: Social representations; Child abuse; Breeding; Family; Education

Recibido: 11/12/2020 Aceptado: 12/11/2020

Correspondencia: (*) nicolasparra@asociacioneducar.com 


\section{INTRODUCCIÓN}

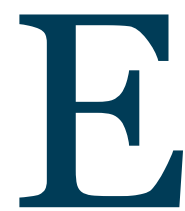

I presente artículo muestra los resultados de la investigación que se evidencian en las representaciones de índole social en poblaciones de menores que han sufrido de maltrato infantil en la Ciudad de Medellín, Colombia, dando cuenta de que, los procesos de crianza de los niños y niñas de la comuna cuatro de la ciudad de Medellín tienen muchos argumentos psicosociales y psicoeducativos que ofrecer para entender cómo es el desarrollo de niños, adolescentes y jóvenes que viven en contextos de violencia.

En atención a la pregunta orientadora ¿Cuáles son las representaciones sociales que hay en torno al maltrato infantil encontrado en los procesos propios de crianza de los niños y adolescentes de la comuna 4 en la Ciudad de Medellín? Los resultados o hallazgos se presentan en torno a los objetivos de la investigación, que pretenden develar cuales son las representaciones sociales alrededor del maltrato infantil, revisando las creencias con las que se configuran en los procesos de crianza y cuáles son las ideas de maltrato, abuso emocional, físico, sexual, la negligencia y el abandono.

De esta manera se marcan los derroteros para entablar un proceso investigativo serio y concienzudo que aporte la suficiente ilustración para en un momento determinado ofrecer herramientas que desemboquen en la transformación de realidades políticas concretas y respondan a los aspectos misionales de la academia en la reproducción social del conocimiento y la conexión directa con la sociedad civil.

\section{MARCO TEÓRICO}

Tras el rastro de la violencia

El fenómeno de la violencia es una actividad que tiene principios que deben rastrearse hasta las más primitivas conductas de agresión y defensa vistas en los organismos más simples, por lo que, debe comprenderse la violencia a la luz de los dispositivos básicos de aprendizaje y claro está, desde una perspectiva que vaya de lo evolutivo a lo psicosocial.
A lo largo de la historia, la cultura y la idiosincrasia han generado diversas prácticas de crianza que han respondido a unas necesidades específicas de cada época. Ese conocimiento del sentido común se convierte en una prescripción de la conducta de los padres y cuidadores, luego aparecen los avances científicos de la medicina, la pedagogía, la psicología y el desarrollo humano, como alternativa para validar o reprobar esas prácticas generadas por un consenso social.

La aprobación de prácticas de crianza maltratantes en nuestra sociedad por parte de los padres es como una suerte de acuerdo implícito que emergió en los grupos de discusión evaluados, donde se encontró que las ideas y creencias que se construyen y se validan en la interacción vienen de lo particular a lo macro, entendiendo así que la etiología del maltrato está anclada en lo histórico, social y cultural.

Otro asunto, que emerge en la interacción, es el consenso al que los padres llegan a la hora de categorizar el maltrato, se puede decir que socialmente se reconoce en mayor medida el maltrato físico y sexual, pero el abuso emocional y el abandono emocional son menos reconocidos $\mathrm{y}$ a su vez mayormente practicados. "El abuso emocional se considera tanto un factor de toda clase de maltrato como una entidad distinta. Existe un aspecto de traición en todo insulto de un cuidador resultado de la violación de las normas sociales y de la confianza interpersonal.

\section{METODOLOGÍA}

El enfoque metodológico elegido para trazar este camino es el interaccionismo simbólico, que se entiende como la forma de intentar proveer de una explicación rigurosa y sistemática de las conductas de los individuos en términos de las conductas comunitarias y prosociales expresadas dentro del grupo humano o los grupos humanos a los cuales se pertenezca, por lo cual la estrategia metodológica para el análisis y la recolección de la información son los grupos de discusión, al posibilitar la emergencia de las representaciones sociales a través de 
la interacción y conversación con padres y madres de familia de la comuna cuatro de la ciudad de Medellín, sector que ha sido históricamente atropellado por los enfrentamientos de grupos armados al margen de la ley y por problemáticas tanto económicas como psicosociales.

Se recogen datos obtenidos de entrevistas abiertas hechas a un grupo de Padres de Familia de niños y adolescentes pertenecientes a la comuna 4 de la Ciudad de Medellín. Para proteger la identidad de los participantes, se ponen las citas por conjuntos de cuatro letras, las cuales aparecen en mayúscula y se denotan al final de cada cita del discurso de estos Padres de Familia.

Tabla 1. Abreviaturas correspondientes a los ocho Participantes de la muestra.

\begin{tabular}{c}
\hline $\begin{array}{c}\text { Identificación para los } \\
\text { Participantes }\end{array}$ \\
\hline ESJM \\
REZF \\
LHDM \\
RPRF \\
PECF \\
GLYF \\
CMCF \\
FGJF \\
\hline ente: Elaboración propia (2021).
\end{tabular}

Nota. Fuente: Elaboración propia (2021).

Al hablar de representaciones sociales alrededor del maltrato infantil, se concibe la existencia de unos códigos sociales de comportamiento que generan las representaciones sociales sobre el maltrato infantil, entendiendo las representaciones sociales como pauta para la acción.

Un ejemplo de lo anterior, es la creencia que existió durante varios siglos respecto a la decisión de los padres de envolver a los menores en ropajes rígidos, tal práctica fue reprobada por la ciencia validando la importancia de estimular el movimiento en los menores. De esta manera, las representaciones sociales se configuran como guías para la acción.

Es por eso que, los padres deciden como actuar frente a sus hijos, cuando construyen una idea sobre la crianza y sobre el niño y la niña en lo individual, para luego validarlo en lo colectivo. Una de las principales creencias que existe entre ellos, y que se develó en los grupos de discusión, es que los padres son una autoridad para sus hijos y por ello deben de actuar como tal, así ellos no reflexionen frente a lo que eso significa.

"Si un niño no quiere seguir una conducta es por falta de autoridad y hay que ganarse el respeto y la autoridad como sea, si hay que recurrir a la correa, toca porque si no hacen lo que se les da la gana" (ESJM).

"Con los chancletazos es que me he ganado el respeto de mis hijas" (REZF).

"Hoy hay mucho proteccionismo, alcahuetería y permisividad. 3 chancletazos bien dados en las nalgas y listo, ponemos a caminar a mucho malcriado que existe en hogares, calles y colegios. Es que la gran mayoría ya no quieren respetar a padres, madres, profesores, mayores (familiares o vecinos), autoridad policial, entre otras" (LHDM).

En atención al objetivo de develar las representaciones sociales que hay en lo relativo al maltrato infantil identificado en los procesos de crianza de los niños y adolescentes de la comuna 4 en la Ciudad de Medellín, se encuentra que la representación del castigo y el golpe como mecanismo de control que refuerza el respeto a la autoridad, valida la idea de la familia tradicional bajo la cual se establecen normas para regir el comportamiento de los niños, lo cual se replica en el modelo educativo, donde se generan sanciones a todos aquellos que se salgan de los parámetros del comportamiento "normal".

Un ejemplo de esto es la frase recurrente que alude a que "la letra con sangre entra", y que deja explícito que es a partir de los castigos y las penalidades que los sujetos pueden aprender y "ser personas de bien", convirtiendo los procesos de crianza y aprendizaje en una experiencia que se construye desde el miedo a equivocarse, porque de ser así se recibe un golpe, un castigo o no eres validado como sujeto, rompiendo con los vínculos seguros y resilientes que se esperan tejer en los procesos educativos y de crianza. 
"Pues yo sí aprendí a respetar a mis padres con los golpes, pero también crecí teniéndoles pavor y sin poder tenerles confianza. Sigo respetándoles, pero me es muy difícil acercarme a ellos como para alguna confidencia, lo que se llamaría una relación normal de padres e hijos no hay" (RPRF).

"El éxito de los golpes era el miedo que generaban y que por eso los niños y las niñas no repetían los malos comportamientos" (PECF).

Es a partir del establecimiento de esas normas que se fijan las acciones para regular los actos que las incumplan. De esta manera, se configura el castigo como mecanismo de control de los sujetos, aceptando todas las formas de castigo, por perniciosos que sean, para lograr el objetivo de tener una sociedad homogeneizada.

Se puede afirmar que la familia, es el resultado del deber ser de las instituciones, para llegar a unos objetivos estructurales y de capital que exige el modelo económico de explotación capitalista, donde la moral es fundamental para producir unos ciudadanos que se adapten a ese deber ser y así mantener el control, la producción y el orden.

Por ello, aparece la medicalización en la sociedad occidental, para normalizar a aquel que se sale del estándar exigido, y este asunto se extiende a todas las relaciones. Los padres aprendieron a medicalizar al niño, antes de darle afecto.

La medicalización, el castigo, el encierro y la exclusión social son algunos de los mecanismos dedicados al control que se ejerce de forma masiva sobre los individuos que se "desvían" de la normalidad y del orden esperado de una sociedad capitalista. En la siguiente afirmación, se encuentran esa creencia del castigo como solución para provocar que los sujetos sean productivos y encajen en la sociedad.

"Yo recuerdo muchas pelas que me dieron, porque me volaba del colegio, por esto, por lo otro y pienso si no me hubiera corregido que hubiese sido de mí, incluso lo viví en la casa, el hijo calavera o la oveja negra de la familia, él que no hace nada, ese fue al que no le dieron el correazo" (ESJM).
Todos estos elementos, explican de donde surge la idea de castigar, la manera como construyen los padres una concepción legitima del niño y el porqué de la actitud que toman frente a ellos. Para los padres, los niños son "el futuro de la sociedad" esa afirmación en la lógica Foucaultiana se traduciría en que los niños son el futuro de la sociedad capitalista en la que vivimos, por ello emerge la necesidad de controlarlos a como dé lugar para que así, estén listos para producir.

Es a partir de estas creencias, que aparece el castigo y las prácticas de crianza que responden a dicho asunto y que se construyen en lo colectivo. En ese sentido, las representaciones sociales sirven como andamiaje para desarrollar una concepción del niño y para guiar la acción frente a como debe ser su crianza.

Al establecer una concepción del niño y la niña y de lo que debe ser y cómo se debe comportar, también se establecen las prácticas de crianza "maltratantes" consensuadas, que responden a esa necesidad de control de la sociedad capitalista y tienen como objetivo corregir el comportamiento que se salga de ese ideal. Para corregir ese comportamiento, surge el milenario castigo, que es un legado que se ha transmitido generacionalmente, con la idea de castigar a las personas para modificar sus conductas.

Este asunto sigue vigente, generando diversas posturas que validan o rechazan rotundamente el asunto, el rechazo viene de expertos en el tema que han intentado crear conciencia frente a dicha problemática y que, a pesar de ello, no han obtenido la respuesta esperada.

Es así como, señalar conductas como inadecuadas y establecer acciones maltratantes de regulación, generan sensaciones en los sujetos que los guían a actuar a partir de miedos creados, con los cuales las relaciones e interacciones que se establecen con otros sujetos buscan no llegar a situaciones que sean merecedoras de castigos. Por esta razón, se puede evidenciar como, en los padres participantes, surgen discursos que replican el imaginario de la sanción como mediadora de las relaciones entre las personas. 
"Yo recuerdo que en primero de primaria la hermana nos daba reglazos en la mano y así era que entendíamos" (PECF).

Esta afirmación, devela un asunto que los adultos aprendieron en sus hogares y que validaban sus contextos educativos, la escuela se presenta como un escenario de construcción de las representaciones sociales alrededor del castigo, permitiendo así el maltrato como mediador de las relaciones, por esto se puede decir que la escuela castigadora produce una sociedad de castigadores.

Es menester reconocer, que los padres de familia traen unas representaciones legitimadas desde sus hogares y validadas en lo colectivo, sus colegios y sus entornos relacionales, con los cuales establecen criterios para la construcción de prácticas de crianza, las cuales, en este caso, se fundamentan en la continuidad de las sanciones maltratantes como mecanismo de control y toma de conciencia.

Se puede apreciar la intención transformadora de los padres, los niños son la esperanza de la sociedad y se "asume al niño como parámetro para la transformación de nuestras ciudades", pero el ideal transformador, no justifica las acciones ejecutadas de los padres hacia sus propios hijos, que terminan por promover una cultura violenta que se expande en los demás escenarios. Además, ese ideal, es desreponsabilizante porque son los adultos en conjunto con los niños, quienes deben soñarse un futuro justo para las próximas generaciones.

"Mi madre me dio mis correazos cuando tenía 8 porque no quería estudiar, hoy en día soy Ingeniera Civil y diseñadora Gráfica. A los 15 me dio unas cachetadas por llegar tarde y el aprender a estar en casa sin andar de fiesta cada fin de semana me disciplino para el éxito. Aplique la misma crianza para mi hijo tiene 21 años y estoy muy orgullosa de él Así que nadie me diga que un correazo a tiempo no es bueno más vale ver llorar un hijo por un rato que después llorar uno porque es un ser humano problemático. La disciplina no es opcional es tan obligatoria como el amor (GLYF).

La anterior postura, valida que el hogar fue la primera escuela para el castigo, los padres justifican el maltrato que ejecutan con el maltrato que recibie- ron, además justifican las acciones maltratantes como válidas por promover la disciplina. Esto evidencia dos elementos esenciales en la construcción de una representación social, el primero, el nodo central, que es el sistema que da significado a la representación social.

"Cuando ya se le enfrentan a uno y le faltan al respeto ahí si cojo una chancla, alzó la voz o les doy una palmada. Lo que si no estoy de acuerdo con que se les pegue en la cara. Yo trato de no hacer con ellos lo que ellos me cuentan que le hacen a los amigos y que les hace mucho daño" (REZF).

"La correa es importante, hay que usarla adecuadamente sin golpear su cara y sin exageraciones" (CMCF).

"Es muy difícil no gritar, porque controlar ese momento de estrés es muy complejo en la práctica" (FGJF).

Otro aspecto que se evidencia en la narrativa de los padres es que estos no reconocen algunas prácticas como maltrato físico, cuando se les pregunta si una palmada es maltrato físico, manifiestan que para ellos no lo es y que incluso los psicólogos son exagerados en afirmar que cualquier cosa es maltrato, tampoco reconocen los gritos y la indiferencia como maltrato emocional y abandono, respectivamente.

"Yo a mis hijos los he criado con mucho amor, pero también los he regañado y sus nalgadas les he dado. No se puede confundir buena crianza con maltratos físicos ni traumas, por favor una cosa no tiene nada que ver con la otra" (YLMF) "Para mí los psicólogos se tiraron en la crianza y en la educación de los niños al decir que todo es maltrato" (ESJM)

Según la psicóloga Natalia Varela, "seis de cada diez niños entre los 2 y los 14 años son castigados físicamente a nivel mundial". De acuerdo con la especialista, "más o menos el $25 \%$, es decir, un cuarto de la población colombiana acepta el castigo físico como un método de crianza legítimo y válido". "Hay que tener en cuenta que puede haber más casos, que no se registran, porque aún hay cultura de temor a denunciar", explica César Darío Guisao, exdirector del ICBF. (Varela, 2017)

Es claro que hay una delgada línea entre la sanción y el maltrato y esa línea se traspasa a diario, en los 
anteriores datos se reconoce el gravísimo porcentaje de maltrato físico, pero no se reconocen los casos de maltrato emocional, asunto que se develó en las prácticas de crianza de los padres evaluados. Esto es una muestra de la naturalización de la violencia en las familias, que finalmente es una de las causas de la naturalización de la violencia en nuestra sociedad.

Todo lo anterior da cuenta de que las prácticas, conocimientos, actitudes y creencias frente a la crianza, también tienen relación con las características de las familias.

Este panorama de las familias actuales, es un reflejo de las preocupaciones de la sociedad contemporánea que se ha centrado en el tener y no en el ser, en el acumular para responder a unas necesidades vacuas creadas por un capitalismo salvaje, desligándose de las relaciones y del construir con el otro, de potenciar lazos familiares y de movernos desde la solidaridad, el acompañamiento y la compasión.

\section{CONCLUSIONES}

En definitiva, el maltrato infantil está relacionado con las creencias y las representaciones sociales que traen los padres. El núcleo figurativo de estas representaciones se estructura en una sociedad con un modelo de explotación capitalista a la que le interesa tener un control frente a los sujetos y en unas familias que permiten este tipo de control por un marco justificatorio que se inscribe en la crianza que ellos mismos experimentaron, esto se traduce en prácticas perniciosas que afectan el desarrollo integral de la niñez colombiana.

La respuesta a esta problemática estaría enfocada en una educación hacía los padres para fisurar los arquetipos de violencia, orientándose a transformar las representaciones sociales alrededor del maltrato infantil, para que los padres resignifiquen dichas prácticas y sea posible una sociedad menos violenta y más justa. En este sentido, también es importante tener en cuenta a los futuros padres, por lo cual la oportunidad de formarlos estaría en la educación primaria, secundaria y universitaria. Los golpes y los malos tratos en la infancia han fomentado históricamente la violencia, enseñar a vivir en comunidad comienza por el respeto.
Es posible deslegitimar las prácticas violentas a través de diferentes procesos de formación y educomunicación con las familias para humanizar la crianza y movilizarlas desde el respeto, la compasión, la solidaridad, el reconocimiento y trámite de las emociones, el desarrollo de la empatía, el acompañamiento amoroso, la construcción consiente de límites y normas, el fomento del pensamiento crítico, la responsabilización de sí mismos y de su entorno, reconociendo a los niños, niñas y adolescentes como gestores de su propio desarrollo y así darles el lugar que históricamente les ha sido negado. Cuando dejamos de enseñar reglas y comenzamos a estimular criterio, a reflexionar sobre los contextos y las implicancias de nuestros actos, dejamos de entrenar y comenzamos a educar.

Otra respuesta a esta problemática puede estar orientada a la promoción del modelo de crianza denominado "Crianza humanizada" Este modelo sostiene que las relaciones que emergen en los procesos de crianza son determinantes para el futuro emocional y relacional del niño y en estas el buen trato o el maltrato estructuran los diferentes tipos de vínculo.

Finalmente, y apelando por última vez a los presupuestos de la teoría foucaultiana bastará con decir que el hecho de entender críticamente cómo se mueve y desenvuelve un sistema, no implica transformar realidades inmediatamente, pero, permite vivir en un escenario donde la hipocresía ya no es posible. 


\section{REFERENCIAS}

Hillis, S., Mercy, J., Amobi, A., \& Kress, H. (2016). Global Prevalence of Past-year Violence Against Children: A Systematic Review and Minimum Estimates. Pediatrics, 137(3), e20154079. https://doi.org/10.1542/peds.2015-4079

Nguyen, K. H., Kress, H., Villaveces, A., \& Massetti, G. M. (2019). Sampling design and methodology of the Violence Against Children and Youth Surveys. Injury prevention : journal of the International Society for Child and Adolescent Injury Prevention, 25(4), 321-327.

https://doi.org/10.1136/injuryprev-2018-042 916

Chiang, L. F., Kress, H., Sumner, S. A., Gleckel, J., Kawemama, P., \& Gordon, R. N. (2016). Violence Against Children Surveys (VACS): towards a global surveillance system. Injury prevention : journal of the International Society for Child and Adolescent Injury Prevention, 22 Suppl 1(Suppl 1), i17-i22.

https://doi.org/10.1136/injuryprev-2015-0418 20 https://doi.org/10.1136/injuryprev-2018-04 2916

Finkelhor, D., Turner, H., Ormrod, R., \& Hamby, S. L. (2010). Trends in childhood violence and abuse exposure: evidence from 2 national surveys. Archives of pediatrics \& adolescent medicine, 164(3), 238-242.

https://doi.org/10.1001/archpediatrics.2009.2 83

Finkelhor, D., Turner, H. A., Shattuck, A., \& Hamby, S. L. (2013). Violence, crime, and abuse exposure in a national sample of children and youth: an update. JAMA pediatrics, 167(7), 614-621. https://doi.org/10.1001/jamapediatrics. 2013.42

Finkelhor, D., Shattuck, A., Turner, H. A., \& Hamby, S. L. (2014). Trends in children's exposure to violence, 2003 to 2011. JAMA pediatrics, 168(6), 540-546.

https://doi.org/10.1001/jamapediatrics.2013. 5296

Cross, T. P., Mathews, B., Tonmyr, L., Scott, D., \& Ouimet, C. (2012). Child welfare policy and practice on children's exposure to domestic violence. Child abuse \& neglect, 36(3), 210-216.

https://doi.org/10.1016/j.chiabu.2011.11.004 